\title{
In Vitro Bioactivity Test of Senna Alata (L.) Roxb Leaves Extract
}

\author{
Fri Rahmawati ${ }^{1}$, Nur Nunu Prihantini ${ }^{2}$, Bella Christina Hady ${ }^{3}$ \\ 1,2,3 Medical Faculty, Univeristas Kristen Indonesia, Jakarta \\ Corresponding Author: Fri Rahmawati
}

DOI: https://doi.org/10.52403/ijhsr.20220241

\begin{abstract}
Traditionally, Senna Alata (L.) Roxb (Ketepeng Cina) has been used as an alternative medicine for typhus, diabetes, malaria, herpes, eczema, asthma, stomach ulcers, syphilis, influenza, bronchitis, intestinal worms, fungal skin diseases such as tinea versicolor, ringworm and scabies. The study's objective was to determine the active compounds, antioxidant potency and antifungal activity of Senna Alata (L.) Roxb leaves extract. Samples were extracted using the maceration method with $70 \%$ ethanol, ethyl acetate and N-hexane as solvents. Phytochemical analysis used the Harbone method, antioxidant testing using 2,2-diphenyl-1-picrylhydrazyl (DPPH) method and antifungal testing using the agar diffusion method. Phytochemical results proved that all Senna Alata (L.) Roxb leaves extract contained alkaloids, saponins, tannins, flavonoids, triterpenoids, steroids, glycosides. While the antioxidant test stated that ethanol and ethyl acetate solvents had solid antioxidant potential with the Inhibition Concentration $\left(\mathrm{IC}_{50}\right)$ values obtained were $66.01 \mathrm{ppm}$ and $78.23 \mathrm{ppm}$ respectively. While the solvent $\mathrm{N}$-hexane has a moderate antioxidant potential, the $\mathrm{IC}_{50}$ value obtained is $138.80 \mathrm{ppm}$. The diameter inhibition zones of ethanol, ethyl acetate, and N-hexane extracts against the Candida albicans were $8.66 \mathrm{~mm}, 10.22 \mathrm{~mm}$ and $8.84 \mathrm{~mm}$, respectively. Cryptococcus neoformans was only inhibited by ethyl acetate and N-hexane Senna Alata (L.) Roxb leaves extracts with diameter inhibition zones of $4.84 \mathrm{~mm}$ and $4.44 \mathrm{~mm}$, respectively.
\end{abstract}

Keywords: Antifungal, Antioxidant, Candida albicans, Cryptococcus neoformans, Phytochemical, Senna alata (L.) Roxb

\section{INTRODUCTION}

Indonesia is a tropical country with a high level of humidity so that it supports the growth of various plants and microorganisms well; one of the microorganisms that grow well in a tropical climate is a fungus. According to the Centers for Disease Control (CDC), there are millions of species of fungi, but only a few hundred species are capable of infecting humans and causing disease. The most common species infecting humans are Aspergillus, Candida, Trichophyton, Blastomyces, Cryptococcus, Histoplasma, Mucormycetes, and Coccidioides can survive in human hosts [1].
Candidiasis is an infection caused by Candida, either primary or secondary to other diseases, generally occurring in the skin, oral mucosa, gastrointestinal tract, vagina, and penis. Candida has 200 species, but only a few are opportunistic pathogens. Candida albicans is the most common species that causes candidiasis worldwide. C. albicans cause about $60 \%$ of candidiasis [2]. The prevalence of candidiasis is high in developing countries and mainly occurs in tropical areas with high humidity. Candidiasis cases in Indonesia rank third in the incidence of dermatomycosis, with a prevalence of around $20-25 \%$ [3]. 
There are many types of treatment for fungal infections, but only a few groups of drugs can treat mucosal and systemic infections caused by $C$. albicans. Over time, it was found that some Candida species became resistant to azole antifungal drugs. It triggers the development of new antifungal drugs as the main challenge currently continues to overcome antifungal resistance [4]. So it is necessary to develop alternative drugs that are more efficient and tolerant than drugs on the market, which are pretty urgent.

Besides C. Albicans, which infects superficially, some fungi infect systemically, such as Cryptococcus neoformans. Cr. neoformans is a fungus that causes lung disease and can spread to other body parts, such as the brain's lining, both in humans and animals. Infections due to $\mathrm{Cr}$. neoformans are termed cryptococcosis [5]. $C r$. neoformans has characteristics similar to that of C. Albicans, which is opportunistic in that it infects hosts with weak immune systems, such as in hosts with chronic diseases (AIDS, cancer) and immunosuppressive treatments such as corticosteroids. Source of infection $\mathrm{Cr}$. neoformans in nature comes from bird droppings, especially pigeons that are dry and rarely exposed to sunlight. The droppings can be a source of infection if carried with dust through the air and then inhaled by humans.

Fungal infection therapy takes a long time and is still limited, so alternative therapies are needed. Alternative therapy can be in the form of traditional medicine. Traditional medicine has convenience. Namely, the price is lower than conventional medicine and access to available. One of the plants often used as an alternative treatment for fungal diseases is Senna Alata (L.) Roxb [6]. The Senna Alata (L.) Roxb plant is a plant from the leguminosae family that grows in tropical and humid areas like Asia and Africa. Senna Alata (L.) Roxb has a different name in each region in Indonesia, such as tabankun.
Traditionally, Senna Alata (L.) Roxb has been used to treat typhus, diabetes, malaria, asthma, herpes, eczema, constipation, gastric ulcers, syphilis, herpes, influenza, bronchitis, intestinal worms, and especially fungal skin diseases such as tinea versicolor, ringworm and scabies. Several studies have shown that the Senna Alata (L.) Roxb plant has pharmacological activities. It includes antibacterial, antioxidant, antifungal, dermatophytes, anticancer, hepatoprotective, antilipogenic, anticonvulsant, antidiabetic, antihyperlipidemic, antimalarial, antihelmintic, and antiviral. The pharmacological activity of the Senna Alata (L.) Roxb is closely related to the content of the active compounds contained in the plant [7].

$$
\text { Senna Alata (L.) Roxb is an }
$$
alternative medicine for fungal diseases because it contains active compounds that act as antifungals. Senna Alata (L.) Roxb leaves are fungistatic because they contain chrysophanic acid (dehydroxymethylanthraquinone). Tannins, phenols, glycosides, alkaloids, saponins, and anthraquinones. Senna Alata (L.) Roxb stems also has fungistatic properties because it contains 1,5,7-trihydroxy-3-methylanthraquinone (alatinone), free aglycone, kaempferol, -sitosterol and sennoside. Senna Alata (L.) Roxb also has antioxidant activity because it is rich in flavonoids [8].

Based on the community's practical use of the Senna Alata (L.) Roxb plant, the research aimed to determine the class of active compounds, antioxidant activity, and antifungal potential of Senna Alata (L.) Roxb leaves extract against $C$. albicans and $C r$. neoformans. The study aims to determine the active compounds, antioxidant activity, and antifungal potency of various extracts of Senna Alata (L.) Roxb leaves.

\section{LITERATURE REVIEW}

Senna Alata (L.) Roxb is a plant that comes from Argentina, South America. Then spread pantropically, especially in Asia and Africa, one in Indonesia. Indonesia 
is a country with a tropical climate, which has a dry season and a rainy season that allows the growth of wild, abundant and fertile Senna Alata (L.) Roxb plants. Senna Alata (L.) Roxb plants usually grow in lowland to mountainous areas 1,400 meters above sea level [9]. Ketepeng plants in Indonesia have various regional names, such as kupang leaves (Malay), ringworm leaves, ura'kap (Sumatra), ngganggang (Tapanuli), ki manila, ketepeng gede, katepeng (Sunda), Chinese Ketepeng, ketepeng kebo, ketepeng rhino (Central Java), acong-acong (Madura), tabakum (Tidore), and kupang-kupang (Ternate). The foreign names for the ketepeng plant are seven golden candlesticks (English), candle bush, crawcraw plant, Acapulco, ringworm bush, or ringworm plant chum-het-thet (Thailand), and dui ye dou (China).

The Senna Alata (L.) Roxb plant is classified as a dicotyledonous plant with a taproot system, i.e. the root continues to grow into the primary root and has smaller branches, which function to expand nutrient absorption and strengthen the erection of the stem. The stem is round, can grow about 3-4 meters, has a sympodial branching system, has yellowish-green leaves with a slightly wide shape with a length of $50-80 \mathrm{~cm}$, bright yellow flowers, and brownish yellow fruit that reaches $25 \mathrm{~cm}$ long: unpleasant odour, spicy taste and warm when consumed. The seeds are considered an alternative to nuts because of their high protein and carbohydrate content [10].

Senna Alata (L.) Roxb lives in open and humid areas, usually growing on roadsides, riverbanks, waterways, forests, bushes, meadows, lakesides, and ponds. Senna Alata (L.) Roxb grows best in full sun and moisture [11]. Grows well in fertile to sandy, acidic to slightly alkaline soils, with good drainage; with a $\mathrm{pH}$ ranging from 5.6 to 7.8. This plant is reported to grow in areas with an annual rainfall of 600-4300 $\mathrm{mm}$ and an average annual temperature of 15-30 ${ }^{\circ} \mathrm{C}$. The metabolites contained in Senna Alata (L.) Roxb are tannins, alkaloids, flavonoids, terpenes, anthraquinones, saponins, phenolics, cannabinoid alkaloids. Besides, 1,8-cineole, caryophyllene, limonene, -selinene, -caryophyllene, germacrene D, cinnamic acid, pyrazole-5ol, methaqualone, isoquinoline, quinones, reducing sugars, steroids, and essential oils. Metabolites are more often found in the leaves [12].

Senna Alata (L.) Roxb has antifungal activity, especially fungi that cause skin infections in humans, such as A. niger, $A$. flavus. A. candidus, $P$. patulum, $C$. albicans and $R$. stolonifer, T. mentagrophytes, T. tubrum, $M$. gypseum and $M$. canis. However, the antifungal activity of Senna Alata (L.) Roxb was higher in dermatophytes than in non-dermatophytes. The secondary metabolites of Senna Alata (L.) Roxb are mainly tannins, saponins, flavonoids, and alkaloids. The traditional use of Senna Alata (L.) Roxb leaves by the community is to boil the water and then drink it, or it can be ground and then added a little water and then rubbed on the infected skin surface area. Senna Alata (L.) Roxb leaves are often used by Indonesian people, especially South Sulawesi, to treat fungal skin diseases by mashing and applying directly to infected skin; it has been proven to be effective in relieving itching caused by fungi [13].

Several studies showed that the Senna Alata (L.) Roxb extract showed antibacterial activity against Gram-positive and harmful bacteria, namely Staphylococcus aureus, Bacillus subtilis, Bacillus cereus, Shigella boydii, Shigella dysenteriae, Pseudomonas aureus, Vibrio mimicus, Salmonella paratyphi, Vibrio parahaemolyticus, Salmonella typhi, Vibrio cholera, Streptococcus sp. and Escherichia coli. Senna Alata (L.) Roxb extract showed antibacterial activity close to penicillin, chloramphenicol, fluconazole, and ciprofloxacin. It is due to the secondary metabolite compounds possessed by Senna Alata (L.) Roxb, especially tannins, phenolics, and flavonoids [14]. Previous studies have shown that Chinese Ketepeng extract has anti-inflammatory activity. The 
results showed that $1 \mathrm{mg}$ of Senna Alata (L.) Roxb extract could inhibit cytokines and $\mathrm{T}$ lymphocytes so that the production of TNF$\alpha$ decreased. Tumour Necrosis Factor Alpha (TNF- $\alpha$ ) is a cytokine produced mainly by monocytes or macrophages and is also produced by mast cells, endothelial cells, nervous tissue and lymphocytes such as $\mathrm{T}$ and B lymphocytes and natural killer cells. TNF- is a pro-inflammatory mediator, the higher TNF- levels in the wound, indicating an ongoing inflammatory process. When an injury occurs, inflammation will occur. Inflammation has a function to remove nonvital tissue and prevent infection [15]. Nhexane Senna Alata (L.) Roxb leaves extract showed strong anti-inflammatory potential by significantly reducing rat knee swelling, and N-hexane Senna Alata (L.) Roxb leaves extract was also able to relieve diarrhoea and toothache [16].

Several studies have reported that the biological activity of Senna Alata (L.) Roxb L. Crude extract of the leaves of Senna Alata (L.) Roxb has a robust antioxidant activity with an $\mathrm{IC}_{50}$ value of $2.27 \mathrm{~g} / \mathrm{mL}$. It is because Senna Alata (L.) Roxb contains flavonoid compounds, especially in the leaves. With the presence of antioxidants in Senna Alata (L.) Roxb, this has the potential that Senna Alata (L.) Roxb can be an alternative treatment for diabetes mellitus. Oxidative stress can damage body tissues which can cause diabetes mellitus [17].

Research on mice with diabetes due to streptozotocin induction was given ethanol extract of Senna Alata (L.) Roxb leaves. The results showed a significant increase in the antioxidant system in the liver and kidneys of the mice, namely vitamin $\mathrm{E}$, catalase, glutathione peroxidase and glutathione-s-transferase. In addition, there were several changes in typical biochemical parameters such as protein, urea, uric acid, and creatinine levels from abnormal to regular in control mice and induced mice. It indicates that the Senna Alata (L.) Roxb leaves extract has shown its inhibitory effect on unnecessary protein degradation due to decreased cell damage caused by free radicals. From the above observations, it is clear that Senna Alata (L.) Roxb has helped regulate oxidative stress in diabetic rats, which could significantly support the hypoglycemic potential of Senna Alata (L.) Roxb. Besides being a potential alternative treatment for diabetes, the antioxidant properties of Senna Alata (L.) Roxb have the potential to prevent cancer [18].

The results of studies have been carried out using the hydro-methanol extract from Senna Alata (L.) Roxb in mice to see the degranulation ability of mast cells showed anti-allergic activity through mast cell stabilization and inhibition of lipoxygenase enzymes. The active ingredients in Senna Alata (L.) Roxb can be a potential alternative treatment for allergic diseases such as itching, urticaria and asthma. $C$. albicans is the most common fungal pathogen in humans. C. Albicans is an opportunistic, harmless, and commensal pathogen in approximately $50 \%$ of individuals. $C$. albicans is a normal microflora in the mucous membranes of the oral cavity, respiratory tract, digestive tract and genital organs and its growth is controlled by the immune system and protective bacteria on the intestines and other mucosal surfaces [19].

Cryptococcus is an invasive fungus transmitted by inhalation of spores. It causes cryptococcosis, an infection commonly associated with immunosuppressed individuals with diseases such as AIDS, diabetes, chronic liver disease, chronic kidney disease, prolonged use of steroids, and patients undergoing organ transplantation usually associated with the development of cryptococcal disease [20].

The genus Cryptococcus consists of 37 species and belongs to the phylum Basidiomycota. Of the many species, only two cause cryptococcosis, namely Cryptococcus neoformans and Cryptococcus gattii. Cr. neoformans is divided into three varieties; $C r$. neoformans var. grubii (serotype A), Cr. neoformans 
var. neoformans (serotype D) and $\mathrm{Cr}$. neoformans var. gattii (serotypes $\mathrm{B}$ and $\mathrm{C}$ ). The most common isolate causing infection in humans was $\mathrm{Cr}$. neoformans var grubby serotype A. Varieties Cr. neoformans are distinguished by their biochemical properties and the antigenic composition of the capsular polysaccharide [21].

Cr. Neoformans most commonly infect through spores inhaled by the host and then captured by alveolar macrophages. The host response to cryptococcal infection involves the response of helper $\mathrm{T}$ cells with cytokines including tumour necrosis factor (TNF), interferon- $\gamma$, and interleukin-2, which result in granulomatous inflammation that kills and inhibits $C r$. Neoformans. The capsule protects the fungus from phagocytosis and oxidative injury along with melanin [22].

\section{RESEARCH METHOD}

This research is an experimental study to determine the class of active compounds (phytochemical analysis) and bioactivity (antioxidant and antifungal) from ethanol extract, ethyl acetate and N-hexane from Senna Alata (L.) Roxb leaves. The research was conducted from January to March 2021 at the Parasitology Laboratory, Faculty of Medicine, Christian University of Indonesia and the Research Institute for Spices and Medicinal Plants (BALITTRO) Bogor-West Java. The test material in this study was the leaves extract of Senna Alata (L.) Roxb, which was obtained from the garden of the Research Institute for Spices and Medicinal Plants, Bogor, West Java. Then it was determined at the Research Institute for Biology, Herbarium Bogoriense Bogor Indonesian Institute of Sciences. While the isolates of $C$. albicans and $C r$. neoformans used were obtained from the Parasitology Laboratory of the Faculty of Medicine, Christian University of Indonesia. The number of replications from each treatment group was calculated using the Federer formula. The treatment group consisted of 5 groups consisting of $50 \%$ ethanol extract, ethyl acetate and $\mathrm{N}$-hexane from Senna Alata (L.) Roxb leaves, a negative control group using aqua dest solution and a positive antifungal control using fluconazole. Determination of repetition is determined by the Federer formula, as follows:

Federer formula : $(n-1)(k-1) \geq 15$

Description $: n=$ number of repetitions

$\mathrm{k}=$ number of grouping $=5$

$(\mathrm{n}-1)(5-1) \geq 15$

$(\mathrm{n}-1)(4) \geq 15$

$4 n-4 \geq 15$

$4 \mathrm{n} \geq 19$

$\mathrm{n} \geq 4,75$

Based on these calculations, the result of the sample size is 4.75 . To avoid errors, they were rounded to 5. So each treatment group was repeated five times using Saboraud Dextrose Agar (SDA) which had been cultured by Candida albicans and Cryptococcus neoformans. The tools used in this research are autoclave, rotary evaporator, blender, ose, sterile cotton swab, handscoen, mask, cotton, tissue, cloth, sterile petri dish, marker, Erlenmeyer flask, chemical funnel, glass bottle, test tube, dropper, tweezers, syringe, Bunsen, lighter, analytical balance, stir bar, calliper, and paper disc. The materials used in this study were cultures of $C$. albicans and Cr. neoformans, Senna Alata (L.) Roxb leaves extract in $70 \%$ ethanol, technical ethyl acetate, technical N-hexane, Sabouraud Dextrose Agar (SDA) media, chloramphenicol antibiotics, Chrom agar, sterile distilled water. $0.9 \%$ sterile $\mathrm{NaCl}, 0.5$ McFarland standard, and $10 \% \mathrm{KOH}$. Procedure The research was carried out through several stages as follows: 1) Making simplicia and extract of Senna Alata (L.) Roxb leaves; 2) Phytochemical analysis by Harborne Method, through identification of alkaloid saponins, tannins, flavonoids, triterpenoids/steroids, and glycosides; 3) antioxidant test by DPPH Method; 4) Antifungal activity test through sterilization, manufacture of Sabouraud Dextrose Agar (SDA) media, fungal 
rejuvenation, and antifungal testing by diffution agar method.

\section{RESULT AND DISCUSSION}

The phytochemical analysis is a qualitative measurement method to determine the secondary metabolites' class in plants. The phytochemical analysis included alkaloids, saponins, tannins, flavonoids, triterpenoids, steroids, glycosides. The results of the phytochemical analysis of the Senna Alata (L.) Roxb leaves extract can be seen in Table 1.

Table 1. Results of Phytochemical Analysis of Senna Alata (L.) Roxb Leaves Extract

\begin{tabular}{|l|l|l|l|}
\hline \multirow{2}{*}{ Test } & Extract & \multicolumn{2}{l|}{} \\
\cline { 2 - 4 } & Ethanol & Ethyl acetate & N-hexane \\
\hline Alkaloids & & & \\
- Mayer & + & + & + \\
- Wagner & + & + & + \\
- Dragendor & + & + & + \\
\hline Flavonoids & + & + & + \\
\hline Tannins & + & + & + \\
\hline Saponins & + & + & + \\
\hline Triterpenoids & + & + & + \\
\hline Steroids & + & + & + \\
\hline Glycoside & + & + & + \\
\hline Description: (+) contains the class of compounds tested \\
\hline
\end{tabular}

The results of the phytochemical analysis showed that all Senna Alata (L.) Roxb leaves extracts with $70 \%$ ethanol, ethyl acetate, N-hexane as solvents contained alkaloids, saponins, tannins, flavonoids, triterpenoids, steroids, glycosides.

Antioxidant activity is the ability of a compound or extracts to inhibit oxidation reactions which the percentage of inhibition can express. The parameter used to show antioxidant activity is Inhibition Concentration $\left(\mathrm{IC}_{50}\right)$, the concentration of an antioxidant substance that can cause $50 \%$ of DPPH to lose its radical character or the concentration of an antioxidant substance that gives $50 \%$ inhibition. The $\mathrm{IC}_{50}$ value was determined by calculating the regression analysis of \% inhibition of the Senna Alata (L.) Roxb leaves extract concentration. The results of the antioxidant activity of the Senna Alata (L.) Roxb leaves extract using the DPPH method in $70 \%$ ethanol, ethyl acetate and N-hexane as solvents can be seen in Figure 2 .

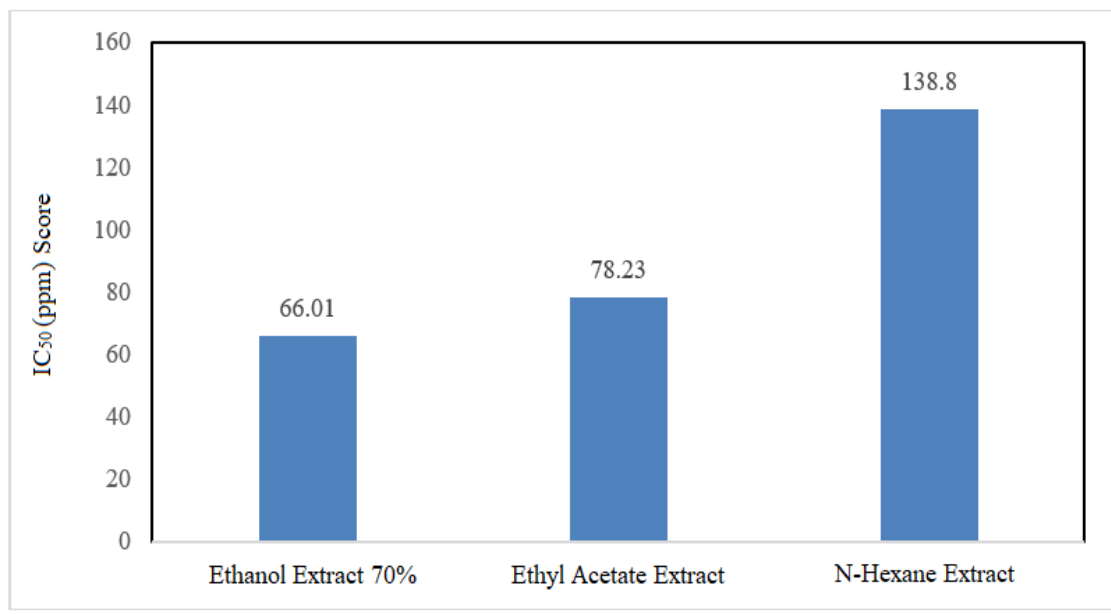

Figure 1. Antioxidant Test Results of Various Senna Alata (L.) Roxb Leaves Extracts

Based on Figure 1, it is known that the Senna Alata (L.) Roxb leaves extract has antioxidant activity. Ethanol and ethyl acetate solvents have strong antioxidant potential, with $\mathrm{IC}_{50}$ values obtained are $66.01 \mathrm{ppm}$ and $78.23 \mathrm{ppm}$, respectively. While the solvent $\mathrm{N}$-hexane has a moderate antioxidant potential, the $\mathrm{IC}_{50}$ value obtained is $138.80 \mathrm{ppm}$.
The antioxidant test data obtained from Senna Alata (L.) Roxb leaves were processed using the Kolmogorov-Smirnov test to show the normality of the data. The inhibition zones in all groups were normally distributed $(\mathrm{p}>0.05)$. The normality test results are used to determine the test method used. Because all variables are normally distributed, the test method used is parametric analysis with the Anova method. 
The Anova test showed a significant difference in antioxidant strength between solvents $(p=0.952)$. The antifungal test method is the diffusion method using disc paper. The formation can see the potential of Senna Alata (L.) Roxb leaves extract as an antifungal of a clear zone around the paper disc, which is an inhibition zone for the growth of $C$. Albicans and $\mathrm{Cr}$. Neoformans. The diameter of the inhibition zone of each Senna Alata (L.) Roxb leaves extract on the growth of $C$. Albicans and Cr. Neoformans can be seen in Figure 2.

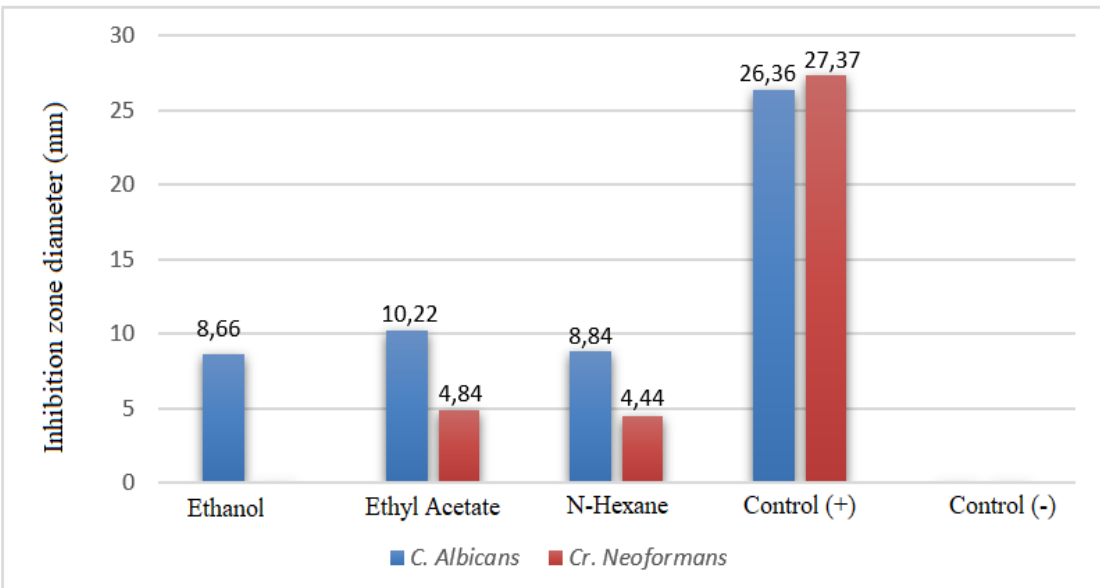

Figure 2. Inhibitory Zone Diameter of Senna Alata (L.) Roxb Leaves Extracts on C. Albicans and Cr. Neoformans

Based on Figure 2, it is known that all Senna Alata (L.) Roxb leaves extracts could inhibit the growth of the fungus $C$. Albicans. The diameter of the inhibition zones of ethanol, ethyl acetate, and $\mathrm{N}$ hexane extracts against the fungus $C$. Albicans were $8.66 \mathrm{~mm}, 10.22 \mathrm{~mm}$ and 8.84 $\mathrm{mm}$, respectively, while the diameter of the inhibition zones of the positive control (fluconazole) against C. Albicans was of $26.36 \mathrm{~mm}$. Antifungal test of Senna Alata (L.) Roxb leaves extracts against $C r$. Neoformans only showed inhibition by ethyl acetate, and N-hexane extracts with inhibition zone diameters of $4.84 \mathrm{~mm}$ and $4.44 \mathrm{~mm}$, respectively. The diameter of the inhibition zone produced by the positive control of fluconazole against $\mathrm{Cr}$. Neoformans of $27.37 \mathrm{~mm}$.

Data on the antifungal potential of Senna Alata (L.) Roxb leaves against $C$. albicans obtained were processed using the Kolmogorov-Smirnov test to show the normality of the data. The inhibition zones in all groups were normally distributed ( $p>$ 0.05 ). The normality test results are used to determine the test method used. Because all variables are normally distributed, the test method used is parametric analysis with the Anova method. The ANOVA test showed a significant difference in the mean zone of inhibition between groups $(p=0.000)$.

Data on the antifungal potential of Senna Alata (L.) Roxb leaves against $C r$. neoformans were processed using the Kolmogorov-Smirnov test to show the normality of the data. The inhibition zone in the ethyl acetate and $\mathrm{N}$-hexane groups were not normally distributed $(\mathrm{p}<0.05)$, while the inhibition zone of the control group (+) was normally distributed $(\mathrm{p}>0.05)$. The normality test results are used to determine the test method used. Because more variables are not normally distributed, the test method used is non-parametric analysis with the Kruskal-Wallis method. The Kruskal-Wallis method showed a significant difference in the mean zone of inhibition between the extract groups ( $\operatorname{sig}=0.001$ ).

Phytochemical analysis was carried out to identify and identify the secondary metabolite compounds in the Senna Alata (L.) Roxb leaves extract with various solvents. The solvents used in this study were $70 \%$ ethanol, ethyl acetate, and $\mathrm{N}$ hexane. The use of several solvents in the 
extraction process is based on the difference in the level of polarity possessed by the three solvents used to extract the active compounds in Senna Alata (L.) Roxb leaves. Ethanol is polar, ethyl acetate is semi-polar, and N-hexane is non-polar. The difference in the polarity level of each solvent used in the extraction affects the secondary metabolites dissolved in the extract [23].

Secondary metabolites are organic compounds resulting from plant metabolism that do not have direct functions in photosynthesis, growth or respiration, solute transport, translocation, protein synthesis, nutrient assimilation, differentiation, formation of carbohydrates, proteins, and lipids. Secondary metabolite compounds are not essential for these plants' life processes and growth but function in certain activities, for example, functioning as emergency nutrients for the survival of these plants. Secondary metabolites have many benefits for the benefit of human life, for example, in the fields of food (colourants, flavours, preservatives, etc.), health (antioxidants, anticancer, antimalarial), environment (anti mosquitoes, anti-weeds, etc.), agriculture (alelopathy, attractants, etc.), and so on) [24; 25]. Based on the results of the phytochemical analysis in Table 1, it can be seen that all of the Senna Alata (L.) Roxb leaves extracts contain secondary metabolites in the form of alkaloids, saponins, tannins, phenolics, flavonoids, triterpenoids, steroids, and glycosides.

Several phytochemical tests on Senna Alata (L.) Roxb with different dosage forms showed somewhat different results. Phytochemical tests of Senna Alata (L.) Roxb simplicia showed the presence of flavonoid, alkaloid, anthraquinone, saponin and tannin compounds. The ethanolic fraction of Senna Alata (L.) Roxb leaves extract contains alkaloids, flavonoids, tannins, saponins and terpenoids, while the chloroform fraction contains tannins, saponins, alkaloids, and flavonoids, and the n-hexane fraction contains alkaloids, saponins, tannins, and terpenoids. Another study found that the ethanolic extract of
Senna Alata (L.) Roxb had active metabolites of flavonoids, alkaloids, saponins, tannins, and terpenoids [24].

Alkaloids are one of the secondary metabolites found in plants, which can be found in the leaves, twigs, seeds, and bark. Alkaloid compounds have many pharmacological effects, namely antiinflammatory, antimicrobial, hepatoprotective, anticancer and increase the antioxidant effect of cells. Alkaloids affect the health sector in the form of triggering the nervous system, raising blood pressure, reducing pain, antimicrobials, sedatives, drugs for heart disease and others. In plants, saponins are evenly distributed in their parts, such as roots, stems, tubers, leaves, seeds and fruits. The content of saponins was more found in young plants than in old plants. Saponins have pharmacotherapy effects, namely anti-inflammatory, vasoprotective, gastroprotective, hemolytic, anticancer and antimicrobial. In addition, they also function as antihypercholesterolemia. Saponins reduce body cholesterol by preventing reabsorption and increasing excretion. Binding saponins obtain anti hypercholesterolemia with cholesterol and bile acids [26].

Flavonoid compounds are phenolic compounds. Flavonoids are now considered indispensable components in various nutraceutical, pharmaceutical, drug and cosmetic applications because pharmacologically, they function as antiinflammatory, antioxidant, antibacterial, antifungal, antiviral, and anticancer. Flavonoids specifically function as antioxidants that can increase self-defence from diseases induced by free radicals. The antioxidant properties of flavonoids can prevent diseases such as cancer, Alzheimer's, atherosclerosis, and others. Tannins are phenolic compounds. Tannins are divided into two groups, namely hydrolyzed tannins and condensed tannins. Tannins have properties as antiinflammatory, antibacterial, antifungal, antidiarrheal, antioxidant. Condensed tannins have other pharmacological effects 
as hypocholesterolemic. Antidiarrheal and antibacterial properties because when tannins meet the intestinal mucosa, they will have a spasmolytic effect that shrinks the intestines to reduce intestinal peristalsis. However, this spasmolytic effect may also shrink the bacterial cell wall or cell membrane, thereby interfering with the permeability of the cell itself. Due to the disruption of permeability, cells cannot carry out living activities so that the growth of living cells is inhibited or even dies [27].

Triterpenoid compounds are compounds whose carbon skeleton is derived from isoprene units and biosynthetically derived from the acyclic C30 hydrocarbon, namely squalene. Triterpenoid compounds have certain physiological activities, such as antifungal, antibacterial, antiviral, anticancer, overcoming liver damage, overcoming menstrual disorders, and overcoming diabetes. Steroid compounds are a class of lipids derived from saturated compounds and are insoluble in water. Steroids have broad pharmacological effects, including hormone regulators, antioxidants, antiasthmatics, bronchodilators and normalized blood. The biological activity of steroids is to increase or control reproduction in humans, for example, estradiol progesterone. Steroid compounds are also used in medicine as cardiotonic, vitamin D precursor, oral contraceptive and antiinflammatory [28].

Glycosides are natural compounds consisting of two parts, namely sugars (glycones) and non-sugars (aglycones). The function of glycosides for humans is as a means of treatment, including cardiac drugs, laxatives, local irritants, analgesics and surface tension lowering agents how glycosides work as cardiac drugs by affecting the sodium pump mechanism. In this case, cardiac glycosides control the body's electrolyte balance (potassium, sodium and calcium ions) in heart cells [29]. The properties of this drug are to maintain heart tone, increase adrenergic nerve tone, and maintain circulating blood volume.
Thus, contraction and heart rate will increase. Free radicals are highly reactive molecules because they have an unpaired electron in their outer orbital. Free radicals have unpaired electrons, so they are reactive unstable and will continue to look for their electron pair. These radicals can form a chain reaction in the body to form new free radicals that cause continuous and continuous cell damage. Free radicals can be neutralized with antioxidants. Antioxidant compounds can donate one or more electron molecules to free radicals so that these molecules become stable again and do not cause cell damage.

One of the plants with the potential as an antioxidant is Senna Alata (L.) Roxb. The antioxidant activity of Senna Alata (L.) Roxb leaves can be carried out in vitro using 1,2-dephenyl2-picrylhydrazine (DPPH) method. DPPH is a free radical that is stable at room temperature, so it is used to assess antioxidant activity. If all the electrons in DPPH are paired and turn into a stable molecule, the colour of the solution will change from dark purple to yellow. The colour change that occurs is due to the presence of compounds that can give hydrogen radicals to DPPH radicals so that they are reduced to DPPH-H. The antioxidant activity of the sample can be assessed from the absorbance value using a spectrophotometer with a wavelength of 517 $\mathrm{nm}$. The parameter used to show antioxidant activity is Inhibition Concentration ( $\mathrm{IC}_{50}$ ) which is the concentration of an antioxidant substance that causes a loss of $50 \%$ of DPPH activity, causing DPPH to lose its radical character. The smaller the $\mathrm{IC}_{50}$ value, the higher the antioxidant activity. Classification of antioxidants is divided into 5 , namely $<50 \mathrm{ppm}$ (very strong), 50-100 ppm (strong), 100-150 ppm (moderate), $150-200 \mathrm{ppm}$ (weak), and >200 ppm is very weak. It happens because the DPPH radical is reduced when it encounters antioxidant compounds. The more antioxidant compounds possessed by the Senna Alata (L.) Roxb leaves extract, the lower the 
absorbance value of these antioxidant compounds [30].

Based on the graph in Figure 1, it is known that the Senna Alata (L.) Roxb leaves extract has antioxidant activity. Based on the $\mathrm{IC}_{50}$ value of each tested Senna Alata (L.) Roxb leaves extract, the ethanol and ethyl acetate of the Senna Alata (L.) Roxb leaves extract had strong antioxidant potential with $\mathrm{IC}_{50}$ values in the range of 50$100 \mathrm{ppm}$, while the $\mathrm{N}$-hexane extract of the Senna Alata (L.) Roxb leaves had moderate antioxidant potential. Because it has an $\mathrm{IC}_{50}$ value in the range of $100-150 \mathrm{ppm}$, this is following previous research, which stated that the crude extract of Senna Alata (L.) Roxb showed antioxidant activity with an $\mathrm{IC}_{50}$ value of $73.57 \mathrm{ppm}$. Based on this, the crude extract of the Senna Alata (L.) Roxb leaves is classified as a moderate or moderate antioxidant because it is in the range of $100-150 \mathrm{ppm}$. Based on the $\mathrm{IC}_{50}$ value of each Senna Alata (L.) Roxb leaves extract, it is known that the ethanol extract of the Senna Alata (L.) Roxb leaves extract has a lower $\mathrm{IC}_{50}$ value than the other two extracts. Because it has the smallest $\mathrm{IC}_{50}$ value, the antioxidant activity is proportional to the $\mathrm{IC}_{50}$ value. Then a statistical test was performed using a Kolmogorov Smirnov (Appendix 6.) The $\mathrm{IC}_{50}$ value for the treatment of ethanol, ethyl acetate, and $\mathrm{N}$-hexane extracts gave a value $(\mathrm{p}>0.05)$, indicating no significant difference between the three extracts.

The difference in antioxidant activity in the extract was thought to be due to the difference in solvent polarity. Antioxidant activity is highly dependent on the type of solvent used because compounds with different polarities show different levels of antioxidant activity.76 Senna Alata (L.) Roxb leaves extract with ethanol solvent showed the highest antioxidant activity, presumably because there were many polar bioactive compounds in the samples of Senna Alata (L.) Roxb leaves. Such as flavonoids. Following the principle of like dissolves like, a compound will dissolve in a solvent with the same properties, so that polar ethanol solvents will attract polar bioactive compounds. Based on the phytochemical analysis of each Senna Alata (L.) Roxb leaves extract, it is suspected that secondary metabolites that have the potential as antioxidants in Senna Alata (L.) Roxb leaves are active compounds of the flavonoid alkaloid and saponin groups. Alkaloids can efficiently stop free radical chain reactions. Saponin compounds can reduce superoxide by forming hydroperoxide intermediates to prevent biomolecular damage by free radicals, while flavonoids are phenolic compounds. The antioxidant activity of phenolic compounds is formed due to the ability to form phenoxide ions which can donate one electron to free radicals [31].

The antifungal potential of the antifungal extract of the Senna Alata (L.) Roxb leaves was determined from the clear zone around the paper disc containing the extract. Based on Figure 2, it is known that all Senna Alata (L.) Roxb leaves extracts were able to inhibit the two test fungi used, except for the ethanol extract of Senna Alata (L.) Roxb, which was unable to inhibit the growth of $\mathrm{Cr}$. neoformans. The classification of fungal growth inhibition responses can be seen in Table 2 .

Table 2. Classification of Fungal Growth Inhibitory Responses \begin{tabular}{|l|l|}
\hline Clear Zone Diameter & Fungal Growth Inhibition Response \\
\hline
\end{tabular} \begin{tabular}{l|l}
$>20 \mathrm{~mm}$ & Very strong \\
\hline $11-20 \mathrm{~mm}$
\end{tabular}

\begin{tabular}{l|l}
$11-20 \mathrm{~mm}$ & Strong
\end{tabular}

6-10 $\mathrm{mm} \quad$ Currently

$\leq 5 \mathrm{~mm}$

The results showed that the Senna Alata (L.) Roxb leaves extract in $70 \%$ ethanol, ethyl acetate, and N-hexane had moderate inhibitory power against $\mathrm{C}$. albicans because it had a clear zone diameter in the range of $6-10 \mathrm{~mm}$. While the Senna Alata (L.) Roxb leaves extract in $70 \%$ ethanol, technical ethyl acetate, and technical N-hexane had a weak inhibitory power against $\mathrm{Cr}$. neoformans because it had a clear zone diameter in the range of 5 $\mathrm{mm}$.

Based on the research results on the antifungal potential of Senna Alata (L.) $R o x b$ leaves extract against $C$. albicans, 
ethyl acetate solvent has the largest inhibition zone compared to ethanol and Nhexane extracts; this is related to the level of the polarity of the solvent used for the extraction process. Senna Alata (L.) Roxb extract with $70 \%$ ethanol solvent produced a clear zone against $C$. albicans of $8.66 \mathrm{~mm}$. The results obtained to follow the results of previous studies, which state that the ethanol extract of the leaves of the Senna Alata (L.) Roxb can inhibit the growth of $C$. albicans. The study using the broth microdilution technique showed that the concentration of the ethanolic extract of Senna Alata (L.) Roxb leaves was $200 \mathrm{mg} / \mathrm{mL} ; \quad 100 \mathrm{mg} / \mathrm{mL} ; \quad 50 \mathrm{mg} / \mathrm{mL}$; $25 \mathrm{mg} / \mathrm{mL} ; 12.5 \mathrm{mg} / \mathrm{mL} ; 6.25 \mathrm{mg} / \mathrm{mL}$ and $3.125 \mathrm{mg} / \mathrm{mL}$ can reduce the growth of $C$. albicans.

Senna Alata (L.) Roxb extract with ethyl acetate as a solvent produced a clear zone against $C$. albicans $10.22 \mathrm{~mm}$. The results obtained to follow the results of previous studies, which stated that the ethyl acetate extract of the Senna Alata (L.) Roxb leaves was known to have antifungal activity with $2.5 \%, 5 \%$ and $10 \%$ against $C$. albicans. Inhibition zones of $7.62 \mathrm{~mm}, 7.66$ $\mathrm{mm}$ and $7.88 \mathrm{~mm}$. Senna Alata (L.) Roxb extract with $\mathrm{N}$-hexane as solvent produced a clear zone against $C$. albicans $8.84 \mathrm{~mm}$. The results obtained to follow the results of previous studies, which stated that the nhexane extract of the leaves of Senna Alata (L.) Roxb had an antifungal activity with concentrations of $10 \%, 30 \%, 50 \%$ showing antifungal activity against $C$. albicans. 10.6 11.3 and $12.6 \mathrm{~mm} \mathrm{[32].}$

Fluconazole antibiotic as a positive control produced a clear zone with an average diameter of $26.36 \mathrm{~mm}$; this value indicates that fluconazole has a very strong inhibitory effect on $C$. albicans because it has a clear zone diameter greater than 20 $\mathrm{mm}$. The use of positive control in experimental research ensures the validity of a study because it is expected to have a positive effect on the test. The results obtained are somewhat different from the results of previous studies, which stated that the ethanolic extract of Senna Alata (L.) Roxb leaves had antifungal activity against Cr. neoformans. In this study using the microdilution method, the minimum inhibitory concentration of the ethanolic extract of Senna Alata (L.) Roxb ranged from $500-1000 \mathrm{~g} / \mathrm{ml}$ at acidic $\mathrm{pH}$. The antifungal activity decreased at neutral $\mathrm{pH}$, and the smallest activity was at $\mathrm{pH} 8$. In addition, the extract was noted to be heat resistant. Differences in research results can be caused by several factors, especially differences in test methods that need to be controlled by researchers. Other factors that can affect the study results are the extraction process of the test sample, disc paper, quality of the test fungus, inoculum size, incubation time, medium, $\mathrm{pH}$ and ambient temperature [33].

The type of solvent used has a major role in the extraction process. The solvent used must have the same polarity as the compound to be drawn. It is presumably because the specific secondary metabolite compounds that act as antifungals were not extracted with ethanol as a solvent so that the secondary metabolites obtained are unable to inhibit the growth of the fungus Cr. neoformans. Senna Alata (L.) Roxb leaves extract with ethyl acetate as a solvent produced inhibition against $\mathrm{Cr}$. neoformans with an inhibition zone diameter of 4.84 mm. While the Senna Alata (L.) Roxb leaves extract with $\mathrm{N}$-hexane as solvent resulted in inhibition against $\mathrm{Cr}$. neoformans with an inhibition zone diameter of $4.44 \mathrm{~mm}$. Based on the diameter of the clear zone produced by the ethyl acetate and N-hexane extract against $C r$. neoformans, it was known that the two Senna Alata (L.) Roxb leaves extracts had low inhibitory properties.

Fluconazole disc paper as a positive control produced a clear zone with an average diameter of $27.37 \mathrm{~mm}$. Fluconazole as a positive control had very strong inhibition against $C r$. neoformans because it had a clear zone diameter of $>20 \mathrm{~mm}$. In this study, the positive control used fluconazole because fluconazole can be used as a treatment for meningitis due to $C r$. 
neoformans, so its inhibitory ability has been clinically tested and can be used as a positive control in this study [34]. The difference in the inhibition between the Senna Alata (L.) Roxb leaves extract with different solvents on the growth of the test fungus was influenced by the type and levels of chemical compounds in each extract. The type of solvent used in the study also affects the compounds present in the extract. The solvent used must have the same polarity as the compound to be drawn.

\section{CONCLUSION}

Based on the study results, it was found that all of the Senna Alata (L.) Roxb leaves extracts contained secondary metabolites of alkaloids, flavonoids, saponins, tannins, triterpenoids, steroids and glycosides. All Senna Alata (L.) Roxb leaves extracts have potential as antioxidants, ethanol extract of Senna Alata (L.) Roxb leaves has the greatest antioxidant power with an $\mathrm{IC}_{50}$ value of $66.01 \mathrm{ppm}$. All Senna Alata (L.) Roxb leaves extracts were able to significantly inhibit the growth of $C$. Albicans with inhibition zones of ethanol, ethyl acetate and N-hexane extracts of 8.66 $\mathrm{mm}, 10.22 \mathrm{~mm}, 8.84 \mathrm{~mm}$, respectively. But $C r$. neoformans was only inhibited by ethyl acetate and N-hexane Senna Alata (L.) Roxb leaves extracts with inhibition zones of 4.84 $\mathrm{mm}$ and $4.44 \mathrm{~mm}$, respectively.

\section{Acknowledgement: None}

\section{Conflict of Interest: None}

\section{Source of Funding: None}

\section{Ethical Approval: Approved}

\section{REFERENCES}

1. Fahliyani, Sara Amiri, Ali Asghar Rastegari, Neelam Yadav, and Ajar Nath Yadav. "Human Fungal Pathogens: Diversity, Genomics, and Preventions." In Recent Trends in Mycological Research, pp. 371394. Springer, Cham, 2021.
2. Yapar, Nur. "Epidemiology and risk factors for invasive candidiasis." Therapeutics and clinical risk management 10 (2014): 95.

3. Widaty, Sandra, Eliza Miranda, Kusmarinah Bramono, Sri Linuwih Menaldi, Melani Marissa, Caroline Oktarina, Danny Surya, and Hening Tirta Kusumawardhani. "Prognostic factors influencing the treatment outcome of onychomycosis Candida." Mycoses 63, no. 1 (2020): 71-77.

4. Fuentefria, Alexandre M., Bruna Pippi, Daiane F. Dalla Lana, Katarzyna K. Donato, and Saulo F. de Andrade. "Antifungals discovery: an insight into new strategies to combat antifungal resistance." Letters in applied microbiology 66, no. 1 (2018): 2-13.

5. Kobayashi, Cláudia Castelo Branco Artiaga, Lúcia Kioko Hasimoto Souza, Orionalda de Fátima Lisboa Fernandes, Sula Cristina Assis de Brito, Ana Cláudia Silva, Efigênia Dantas de Sousa, and Maria do Rosário Rodrigues Silva. "Characterization of Cryptococcus neoformans isolated from urban environmental sources in Goiânia, Goiás State, Brazil." Revista do Instituto de Medicina Tropical de São Paulo 47 (2005): 203-207.

6. Panjaitan, Ruqiah Ganda Putri, and Yohanes Gatot Sutapa Yuliana. "Ethno-Medicinal Plants Used for Medication of Jaundice by The Chinese, Dayak, and Malays Ethnic in West Kalimantan, Indonesia." Pharmacognosy Journal 13, no. 4 (2021).

7. Megawati, Megawati. "Phytochemical Screening, Secondary Metabolites and Biological Activities of Southeast Sulawesi Plants." Jurnal Akta Kimia Indonesia (Indonesia Chimica Acta) (2020).

8. Chatterjee, Saheli, S. Chatterjee, K. K. Dey, and S. Dutta. "Study of antioxidant activity and immune stimulating potency of the ethnomedicinal plant, Cassia alata (L.) Roxb." Med. Aromat. Plants 2, no. 4 (2013).

9. Kartini, Sri. "Lysis Test of Ascaris lumbricoides Eggs After Giving Ethanol Extract of Chinese Ketepeng Leaves (Cassia alata L.)." JPK: Jurnal Proteksi Kesehatan 9, no. 2 (2020): 9-15.

10. Brufau, Gemma, Josep Boatella, and Magda Rafecas. "Nuts: source of energy and macronutrients." British Journal of Nutrition 96, no. S2 (2006): S24-S28.

11. Setiadi, D., M. Susanto, L. Baskorowati, and S. Pudjiono. "Genetic variation of Gmelina arborea Roxb in Trenggalek, East 
Java." In IOP Conference Series: Earth and Environmental Science, vol. 914, no. 1, p. 012014. IOP Publishing, 2021.

12. Clément, Céline, Diego A. Diaz Grados, Bharathi Avula, Ihklas A. Khan, Andrea C. Mayer, Dante D. Ponce Aguirre, Ivan Manrique, and Michael Kreuzer. "Influence of colour type and previous cultivation on secondary metabolites in hypocotyls and leaves of maca (Lepidium meyenii Walpers)." Journal of the Science of Food and Agriculture 90, no. 5 (2010): 861-869.

13. Singh, Ishwar. "Antimicrobials in Higher Plants: classification, mode of action and bioactivities." Chem. Biol. Lett 4, no. 1 (2017): 48-62.

14. Kasmawati, Henny, Ihsan S. Ruslin, Munasari D. Yamin, and Wa Ode Elafita. "Ethnomedicine Studies of Traditional Medicinal Plants of the Muna Tribe in the Village of Bungi Southeast Sulawesi Province of Indonesia." International Journal of Science and Research 8, no. 11 (2019): 1882-7.

15. Kanedi, Mohammad. "Healing Effect Of Leaves Extract Of Candlebush (Cassia Alata L.) On Cutaneous Wound Infected With trichophyton Rubrum." World Journal of Pharmaceutical and Life Sciences 2, no. 5 (2016): 42-50.

16. Zerem, Enver. "Treatment of severe acute pancreatitis and its complications." World Journal of Gastroenterology: WJG 20, no. 38 (2014): 13879.

17. Suryani, Wa Ode Sitti Zubaydah, Muhammad Handoyo Sahumena, Siti Adawia, Ririn Wahyuni, Andi Nafisah Tendri Adjeng, Michrun Nisa et al. "Preparation and characterization of selfnanoemulsifying drug delivery system (SNEDDS) from Moringa oleifera L. and Cassia alata L. leaves extracts." In AIP Conference Proceedings, vol. 2199, no. 1, p. 070011. AIP Publishing LLC, 2019.

18. Lucchesi, Amanda Natália, Natália Tavares de Freitas, Lucas Langoni Cassettari, Sílvio Fernando Guideti Marques, and César Tadeu Spadella. "Diabetes mellitus triggers oxidative stress in the liver of alloxantreated rats: a mechanism for diabetic chronic liver disease." Acta Cirurgica Brasileira 28 (2013): 502-508.

19. Gul-e-Saba Chaudhry, Rehmat Jan, Habsah Mohamad, and Tengku Sifzizul Tengku Muhammad. "Vitex rotundifolia fractions induce apoptosis in human breast cancer cell line, MCF-7, via extrinsic and intrinsic pathways." Research in pharmaceutical sciences 14, no. 3 (2019): 273.

20. Speth, Cornelia, Günter Rambach, Reinhard Würzner, and Cornelia Lass-Flörl. "Complement and fungal pathogens: an update." Mycoses 51, no. 6 (2008): 477-496.

21. Chastain, Daniel B., Andrés F. HenaoMartínez, and Carlos Franco-Paredes. "Opportunistic invasive mycoses in AIDS: cryptococcosis, histoplasmosis, coccidiodomycosis, and talaromycosis." Current infectious disease reports 19, no. 10 (2017): 1-9.

22. Sorrell, T. C. "Cryptococcus neoformans variety gattii." Medical mycology 39, no. 2 (2001): 155-168.

23. Da Silva, Marcelo B., Alexandre F. Marques, Josh D. Nosanchuk, Arturo Casadevall, Luiz R. Travassos, and Carlos P. Taborda. "Melanin in the dimorphic fungal pathogen Paracoccidioides brasiliensis: effects on phagocytosis, intracellular resistance and drug susceptibility." Microbes and infection 8, no. 1 (2006): 197-205.

24. Ngo, Thanh Van, Christopher James Scarlett, Michael Christian Bowyer, Phuong Duc Ngo, and Quan Van Vuong. "Impact of different extraction solvents on bioactive compounds and antioxidant capacity from the root of Salacia chinensis L." Journal of Food Quality 2017 (2017).

25. Illing, Ilmiati, Wulan Safitri, and Erfiana Erfiana. "Uji Fitokimia Ekstrak Buah Dengen." Dinamika 8, no. 1 (2017): 66-84.

26. Kasmawati, Henny, Ihsan S. Ruslin, Munasari D. Yamin, and Wa Ode Elafita. "Ethnomedicine Studies of Traditional Medicinal Plants of the Muna Tribe in the Village of Bungi Southeast Sulawesi Province of Indonesia." International Journl of Science and Research 8, no. 11 (2019): 1882-7.

27. Jeepipalli, Syam PK, Bin Du, Uteuliyev Y. Sabitaliyevich, and Baojun Xu. "New insights into potential nutritional effects of dietary saponins in protecting against the development of obesity." Food chemistry 318 (2020): 126474.

28. Kosaka, Nobuyoshi, Haruhisa Iguchi, Yusuke Yoshioka, Fumitaka Takeshita, Yasushi Matsuki, and Takahiro Ochiya. "Secretory Mechanisms and Intercellular 
Transfer of MicroRNAs in Living Cells." Journal of Biological Chemistry 285, no. 23 (2010): 17442-17452.

29. Chandana, S. "A Study of Certain Ayurvedic Plants Containing Steroidal Saponins with Anti Inflammatory Activity." International Journal of Ayurvedic Medicine 10, no. 3 (2019): 220229.

30. Noack, E. "Cardiac glycosides and intracellular Na", K", Ca"." In Cardiac Glycoside Receptors and Positive Inotropy: Evidence for more than one receptor? Symposium, Munich, October 26-29, 1983, vol. 79, p. 87. Steinkopff, 2013.

31. Angelina, Marissa, Ainul Mardhiyah, Rizna Triana Dewi, Sofa Fajriah, Nusaibah Muthiah, Yasmin Ekapratiwi, Indah D. Dewijanti, and Sri Hartati. "Physicochemical and phytochemical standardization, and antibacterial evaluation of Cassia alata leaves from different locations in Indonesia." Pharmacia 68 (2021): 947.
32. Dawidowicz, Andrzej L., and Małgorzata Olszowy. "Mechanism change in estimating of antioxidant activity of phenolic compounds." Talanta 97 (2012): 312-317.

33. Ngibad, Khoirul, Afidatul Muadifah, Lailatul Jannah Triarini, Laily Rizki Amalia, and Novita Karel Damayanti. "A review of application of natural products as fungicides for chili." Environmental and Toxicology Management 1, no. 2 (2021): 922.

34. Nathan, Vinod Kumar, Mary Esther Rani, Gunaseeli Rathinasamy, Kannan Narayanan Dhiraviam, and Sridhar Jayavel. "Process optimization and production kinetics for cellulase production by Trichoderma viride VKF3." SpringerPlus 3, no. 1 (2014): 1-12.

How to cite this article: Fri Rahmawati, Nur Nunu Prihantini, Bella Christina Hady. In vitro bioactivity test of senna alata (L.) roxb leaves extract. Int J Health Sci Res. 2022; 12(2):304317. DOI: https://doi.org/10.52403/ijhsr. 20220241 